\title{
SOME DIDACTICAL AND SOME PRACTICAL REMARKS ON FREE PLATE WAVES
}

\author{
W. MAYSENHÖLDER
}

Fraunhofer-Institut für Bauphysik, Bereich Akustik, Nobelstrasse 12, D-7000 Stultgart 80, Germany

(Received 27 September 1986, and in revised form 20 February 1987)

\begin{abstract}
It is recalled how wave equations can be generated from dispersion relations. The bending wave equation for thin plates including some higher-order terms is obtained by this general method and compared to the corresponding equation of Timoshenko. Further, the dispersion of free plate waves is derived in a concise and elementary manner without using a scalar potential and a vector potential for the displacements. Finally, as a matter of practical interest, exact and approximate expressions for the ratio of parallel to perpendicular displacements at a plate surface are calculated. Under favourable circumstances measurement of this ratio allows decomposing an observed wave into its quasilongitudinal and bending components.
\end{abstract}

\section{INTRODUCTION}

The present paper has been stimulated by the strange unwillingness of textbooks on elastodynamics and technical acoustics to comment on the question of why the bending wave equation for thin plates (and beams) is fourth-order in the spatial derivatives, while the fundamental equations of elasticity are only second-order. Textbooks on elastodynamics (see, e.g., $[1,2]$ ) and related review articles $[3,4]$ develop the general theory of plates but do not pay much attention, if any, to the limiting case of thin plates. On the other hand, textbooks written mainly for engineers (see, e.g., [5,6]) usually content themselves with the derivation of the bending wave equation for thin plates by means of forces and moments. A notable exception in this respect is reference [7], which treats both points of view; the initial question, however, is not explicitly answered either.

Obviously, there is a gap between fundamental theory and engineering practice, and it is one purpose of this paper to encourage workers in both fields to bridge this gap. First, an elementary, but apparently not widely known answer to the question is provided. It is due to Sauter [8], and his arguments are recalled for convenience and the bending wave equation is derived for thin plates (including some higher-order terms) from the dispersion relation. The subsequent section is devoted to a derivation of dispersion relations of plate waves, which is somewhat simpler and perhaps more transparent than those usually found in textbooks. Finally, the last section may be of interest for practical applications in technical acoustics. The ratio of parallel to perpendicular displacements at the plate surface is worked out for an arbitrary mode and any frequency. Approximate expressions for the two fundamental modes of an infinite plate (which for low frequencies become bending waves and quasi-longitudinal waves) are also given. Below the cut-off frequency for the higher modes these results can be used for a decomposition of an observed wave into its symmetric and antisymmetric components from the measured ratio of parallel to perpendicular displacements. This may serve to illustrate that application of fundamental theory to practical problems may be possible without relevant loss of accuracy and simplicity. 


\section{WAVE EQUATIONS AND DISPERSION RELATIONS}

The spatial and temporal variation of a wavefield quantity $q$, e.g., the particle displacement in a solid, can be expressed as

$$
q=q_{0} \mathrm{e}^{\mathrm{i}(k x-\omega t)},
$$

where $q_{0}$ may depend on the co-ordinates $y$ and $z$, but not on $x$ and $t$. From the relations for space and time derivatives of arbitrary order

$$
\partial^{n} q / \partial x^{n}=(\mathrm{i} k)^{n} q, \quad \partial^{m} q / \partial t^{m}=(-\mathrm{i} \omega)^{m} q
$$

we can construct infinitely many differential equations, e.g., of the form

$$
\mathrm{i}^{m+n} \partial^{m} q / \partial t^{m}-\left(\omega^{m} / k^{n}\right) \partial^{n} q / \partial x^{n}=0,
$$

which are all satisfied by the wave (1).

Usually, however, a differential equation will be called a wave equation only if the coefficients of the derivatives are independent of frequency $\omega$ and wave number $k$. For equations of the form (3) this means that $\omega^{m}$ should be proportional to $k^{n}:$ i.e., it is the dispersion relation $\omega(k)$ which determines the order of the derivatives occurring in the wave equation. If the phase velocity $c=\omega / k$ is constant, then one chooses $m=n$ in equation (3) and obtains

$$
\dot{q}+c q^{\prime}=0 \quad \text { or } \quad \ddot{q}-c^{2} q^{\prime \prime}=0,
$$

where a dot and a prime denote respectively differentiation with respect to $t$ and $x$. In general, $\omega$ may be expanded in a Taylor series,

$$
\omega=\sum_{i=0}^{\infty} \kappa_{l} k^{\prime},
$$

and from equation (2) one is then able to understand Sauter's recipe [8] for how to derive a wave equation from a dispersion relation: replace $\omega$ by i $\partial / \partial t$ and $k$ by $-\mathrm{i} \partial / \partial x$ and operate on the field $q$.

For bending waves in thin plates the phase velocity is approximately proportional to the square root of the frequency or, equivalently, $\omega=\kappa_{2} k^{2}$. Application of Sauter's rule gives

$$
\mathrm{i} \dot{q}+\kappa_{2} q^{\prime \prime}=0
$$

which has exactly the same form as the time-dependent one-dimensional Schrödinger equation for a free particle. This is no surprise, if one recalls that from the free particle energy $\hbar \omega=\hbar^{2} k^{2} /(2 m)$ ( $\hbar=$ Planck's constant divided by $2 \pi, m=$ particle mass) one obtains the same power law for the dispersion relation as for bending waves in the limit of vanishing thickness of the plate. Of course, the quantum mechanical field (the wavefunction) is genuinely complex, whereas the classical field is taken to be complex for mere mathematical convenience.

The imaginary unit, which looks strange in wave equations of classical physics, can usually be avoided by starting from the dispersion relation (5) squared, i.e., $\omega^{2}=\kappa_{2}^{2} k^{4}$ in the example here, leading to the familiar equation

$$
\ddot{q}+\kappa_{2}^{2} q^{\prime \prime \prime \prime}=0
$$

with a fourth spatial derivative. Another reason for choosing equation (7) rather than equation (6) or other equations derived from higher powers of $\omega$ lies in a natural physical interpretation of the individual terms, which in equation (7) correspond to inertial and restoring forces. 
To illustrate the power of this method one may include two more terms in the squared dispersion relation for bending waves,

$$
\omega^{2}=\kappa_{2}^{2} k^{4}\left\{1+d_{1}(h k)^{2}+d_{2}(h k)^{4}\right\},
$$

with dimensionless expansion parameters $d_{1}$ and $d_{2}, h$ being the thickness of the plate. Using the derivatives $\ddot{q}^{\prime \prime}=\omega^{2} k^{2} q \approx \kappa_{2}^{2} k^{6} q+d_{1} \kappa_{2}^{2} h^{2} k^{8}$ and $\dddot{q}=\omega^{4} q \approx \kappa_{2}^{4} k^{8} q$ one can avoid sixth and eighth spatial derivatives and obtain

$$
\ddot{q}+\kappa_{2}^{2} q^{\prime \prime \prime \prime}+d_{1} h^{2} \ddot{q}^{\prime \prime}+\left(d_{2}-d_{1}^{2}\right)\left(h^{4} / \kappa_{2}^{2}\right) \ddot{q}=0 .
$$

An equation of the same type has been derived by Timoshenko [9] for bending vibrations of bars by means of a detailed consideration of forces, moments, shear deformation, rotatory inertia, etc., including an "effective shear modulus" depending on the shape of the cross-section of the bar. It was also Timoshenko [10] who noted that the prefactor of $\ddot{q}^{\prime \prime}$ derived this way does not completely agree with that from the analog to equation (8) for bars. The agreement is rather close, however, if one chooses $8 / 9$ of the ordinary shear modulus for the "effective shear modulus".

Timoshenko's equation for bars [9] is readily transformed into the bending wave equation for thin plates by minor changes in the prefactors (cf. [7], p. 114). It turns out that again the fraction $8 / 9$ is a favourable choice because the expressions corresponding to $d_{1},-(13-4 \sigma) / 48(1-\sigma)$, to $d_{2}-d_{1}^{2}, 1 / 64(1-\sigma)$, and hence to $d_{2},(205-140 \sigma+$ $\left.16 \sigma^{2}\right) / 2304(1-\sigma)^{2}$ ( $\sigma=$ Poisson's ratio), yield results close to those from the exact $d_{1}$ and $d_{2}$, which are given at the end of the following section. The relative deviation is always less than $10 \%$ for all values of $\sigma$ between 0 and $1 / 2$. The prefactor $d_{2}-d_{1}^{2}$ is always smaller than $d_{1}$ by roughly a factor of 15 ; i.e., the last term in equation (9) is clearly less important than the $\ddot{q}$ "-term.

There are variants of the correction terms in equation (9) obtained with numerical factors other than $8 / 9$ (see the paper by Mindlin [11]). By contrast, the derivation of the bending equation of thin plates from the dispersion relation is free from such ambiguities.

\section{ELEMENTARY DERIVATION OF DISPERSION RELATIONS}

The derivation of dispersion relations for free plate waves presented here is elementary in the sense that the displacement field is not split into its irrotational and divergence-free parts. This commonly applied splitting, which is accomplished by introducing a scalar potential and a vector potential, is not compulsory from a mathematical point of view and physically unnecessary because apart from the pure shear waves (where introducing a vector potential would only complicate matters) there are no solutions which are purely irrotational or purely divergence-free.

The fundamental equations of linear elastodynamics for the displacement field $\mathbf{u}$ in an isotropic solid,

$$
\ddot{\mathbf{u}}=c_{t}^{2} \boldsymbol{\nabla}^{2} \mathbf{u}+\left(c_{l}^{2}-c_{t}^{2}\right) \nabla \nabla \cdot \mathbf{u},
$$

with $c_{t}$ or $c_{l}$ the transversal or longitudinal phase velocity are solved by an ansatz for straight waves propagating in the $x$-direction,

$$
\mathbf{u}=\left(\begin{array}{c}
a(y) \\
b(y) \\
0
\end{array}\right) \mathrm{e}^{\mathrm{i}(k x-\omega t)} .
$$


(The plate is assumed to be infinitely extended in $x$-and $z$-directions; its surfaces lie at $y= \pm h / 2$; the less interesting case of pure shear waves with motions only in the $z$-direction is not considered.) This leads to the coupled system of differential equations

$$
(1-\nu) k^{2} a-\nu_{t} a^{\prime \prime}=\left(1-\nu_{t}\right) \mathrm{i} k b^{\prime}, \quad\left(\nu_{t}-\nu\right) k^{2} b-b^{\prime \prime}=\left(1-\nu_{t}\right) \mathrm{i} k a^{\prime}
$$

where

$$
\nu=\left(c / c_{l}\right)^{2}, \quad \nu_{t}=\left(c_{t} / c_{l}\right)^{2}=(1-2 \sigma) / 2(1-\sigma),
$$

and the prime indicates differentiation with respect to $y$ from now on. Symbolically system (12) can be written with differential operators $D_{1}, D_{2}, D_{3}$,

$$
D_{1} a+D_{2} b=0, \quad D_{3} b+D_{2} a=0,
$$

and the ansatz

$$
a=-D_{2} V, \quad b=D_{1} V,
$$

which satisfies equation (13a) identically, reduces the coupled system to an ordinary differential equation of fourth order for the auxiliary function $V$,

$$
(1-\nu)\left(\nu_{t}-\nu\right) k^{4} V+\left(\nu+\nu \nu_{t}-2 \nu_{t}\right) k^{2} V^{\prime \prime}+\nu_{t} V^{\prime \prime \prime \prime}=0,
$$

with the general solution $\left(\nu_{1} \neq \nu \neq 1\right)$

$$
V=C_{1} \mathrm{e}^{\alpha_{1} k y}+C_{2} \mathrm{e}^{-\alpha_{1} k y}+C_{3} e^{\alpha_{2} k y}+C_{4} e^{-\alpha_{2} k y},
$$

where $\alpha_{1}=\sqrt{1-\nu}$ and $\alpha_{2}=\sqrt{1-\nu / \nu_{t}}$. The amplitude "profiles" $a(y)$ and $b(y)$ can now be evaluated from equations (14) and (16).

The boundary conditions for the free surfaces at $y= \pm h / 2$, i.e., vanishing stress components $\sigma_{i y}(i=x, y, z)$,

$$
\sigma_{x y}=\sigma_{y y}=\sigma_{z y}=0
$$

read in terms of $a$ and $b$ and their derivatives at $y= \pm h / 2$ denoted by $a_{ \pm}^{\prime}, b_{ \pm}^{\prime}$, as

$$
a_{ \pm}^{\prime}+\mathrm{i} k b_{ \pm}=0, \quad b_{ \pm}^{\prime}+\left(1-2 \nu_{t}\right) \mathrm{i} k a_{ \pm}=0
$$

Apparently there are two types of solutions, each of which satisfies equations (18) by itself, namely (i) $a(y)=a(-y), b(y)=-b(-y)$ and (ii) $a(y)=-a(-y), b(y)=b(-y)$. The first type is called symmetric and the second antisymmetric because the associated deformations (more exactly the eigenvalues of the strain tensors) are symmetric or antisymmetric with respect to the middle plane $y=0$ of the plate. Thus, with equations (14) and (16) the symmetric solutions are of the form

$$
a_{s}=\mathrm{i}\left[\alpha_{1} C_{2} \cosh \left(\alpha_{1} k y\right)+\alpha_{2} C_{4} \cosh \left(\alpha_{2} k y\right)\right], \quad b_{s}=\alpha_{1}^{2} C_{2} \sinh \left(\alpha_{1} k y\right)+C_{4} \sinh \left(\alpha_{2} k y\right) .
$$

Exchanging cosh and sinh yields the antisymmetric ones:

$a_{a}=\mathrm{i}\left[\alpha_{1} C_{1} \sinh \left(\alpha_{1} k y\right)+\alpha_{2} C_{3} \sinh \left(\alpha_{2} k y\right)\right], \quad b_{a}=\alpha_{1}^{2} C_{1} \cosh \left(\alpha_{1} k y\right)+C_{3} \cosh \left(\alpha_{2} k y\right)$.

(The $C_{i}$ are amplitudes.)

Finally, the condition for non-trivial solutions of equations (18) leads to the dispersion relations, also known as the Rayleigh-Lamb frequency equations,

$$
4 \alpha_{1} \alpha_{2} /\left(1+\alpha_{2}^{2}\right)^{2}=\left[\operatorname{coth}\left(\alpha_{1} k h / 2\right) \tanh \left(\alpha_{2} k h / 2\right)\right]^{ \pm 1},
$$

for symmetric $(+)$ and antisymmetric $(-)$ modes. 
This exercise has served to demonstrate that a derivation of equations (21) can be carried out very easily without introducing scalar and vector potentials for the displacement field. (The auxiliary variable $V$ may be regarded as a kind of potential, but instead of two potentials only one auxiliary function is needed for an elegant solution of the coupled system (13).) Because of its simplicity and transparency the derivation presented here might be worth to be considered for future textbooks.

The complexities of equations (21) will not be discussed here, because this has been done extensively elsewhere (e.g., in references $[1-4,12]$ ). Some approximate expressions are of interest, however, for the fundamental modes in the limit $k h \rightarrow 0$, i.e., for quasilongitudinal waves and bending waves in thin plates. The expansion here is pushed one term further than that of Sauter [8]; subscripts $s$ or $a$ stand for quasi-longitudinal (symmetric) or bending (antisymmetric) waves.

$$
\begin{gathered}
\omega_{s}^{2} \approx c_{p l}^{2} k^{2}\left[1-\frac{\sigma^{2}}{12(1-\sigma)^{2}}(h k)^{2}-\frac{\sigma^{2}\left(6-10 \sigma-7 \sigma^{2}\right)}{720(1-\sigma)^{4}}(h k)^{4}\right], \\
\omega_{a}^{2} \approx \frac{1}{12} c_{p l}^{2} h^{2} k^{4}\left[1-\frac{17-7 \sigma}{60(1-\sigma)}(h k)^{2}+\frac{489-418 \sigma+62 \sigma^{2}}{5040(1-\sigma)^{2}}(h k)^{4}\right],
\end{gathered}
$$

where

$$
c_{p l}^{2}=E / \rho\left(1-\sigma^{2}\right)
$$

with Young's modulus $E$ and mass density $\rho$.

Frequently, an inverse relationship is desired, e.g., wavelength $\lambda$ or phase velocity $c$ as a function of frequency $f=\omega / 2 \pi$. The corresponding equations are then

$$
\begin{gathered}
c_{s}^{2} \approx c_{p l}^{2}\left[1-\frac{\pi^{2} \sigma^{2}}{3(1-\sigma)^{2}}\left(\frac{h f}{c_{p l}}\right)^{2}-\frac{\pi^{4} \sigma^{2}\left(6-10 \sigma-2 \sigma^{2}\right)}{45(1-\sigma)^{4}}\left(\frac{h f}{c_{p l}}\right)^{4}\right], \\
c_{a}^{2} \approx \frac{\pi c_{p l} h f}{\sqrt{3}}\left[1-\frac{\pi \sqrt{3}(17-7 \sigma)}{30(1-\sigma)}\left(\frac{h f}{c_{p l}}\right)+\frac{\pi^{2}\left(3711-3362 \sigma+211 \sigma^{2}\right)}{4200(1-\sigma)^{2}}\left(\frac{h f}{c_{p l}}\right)^{2}\right] .
\end{gathered}
$$

\section{DiSPLACEMENT RATIOS AT THE PLATE SURFACE}

Experimental detection of vibrations of a plate is done by measuring the motion at the surface of the plate. For a careful analysis of such measurements one should know the displacements at the plate surface for all modes involved in the vibration, in particular the ratio $r=a / b$ of parallel to perpendicular displacements at the plate surface (here $y=+h / 2$ is to be considered). This ratio is easily deduced from equations (18) to (20); one gets, for symmetric modes,

$$
r_{s}=\left.\frac{a_{s}}{b_{s}}\right|_{y=h / 2}=\frac{\left(1+\alpha_{2}^{2}\right) \operatorname{coth}\left(\alpha_{1} k h / 2\right)-2 \alpha_{1} \alpha_{2} \operatorname{coth}\left(\alpha_{2} k h / 2\right)}{-\mathrm{i} \alpha_{1}\left(\alpha_{2}^{2}-1\right)},
$$

and for antisymmetric modes

$$
r_{a}=\left.\frac{a_{a}}{b_{a}}\right|_{y=h / 2}=\frac{\left(1+\alpha_{2}^{2}\right) \tanh \left(\alpha_{1} k h / 2\right)-2 \alpha_{1} \alpha_{2} \tanh \left(\alpha_{2} k h / 2\right)}{-\mathrm{i} \alpha_{1}\left(\alpha_{2}^{2}-1\right)} .
$$

These expressions are exact for any modes of the form (11), which describes waves with straight wave fronts, if $\alpha_{1}$ and $\alpha_{2}$ satisfy the corresponding dispersion relations (21) (circular waves behave differently, especially near the origin). With the approximate expressions (22) and (23) one obtains (to lowest order), for quasi-longitudinal waves,

$$
r_{s}^{-1} \approx-\mathrm{i}\{\sigma /(1-\sigma)\} k h / 2
$$


and for bending waves,

$$
r_{a} \approx-\mathrm{i} k h / 2,
$$

reflecting the familiar dominance of longitudinal (transversal) motions in quasi-longitudinal (bending) waves (which is rarely quantified in textbooks).

The lowest-order approximations (28) and (29) for the two fundamental modes also follow from the boundary conditions (18) if the lowest non-trivial approximations for $a$ and $b$ are inserted. For the quasi-longitudinal mode $a_{s}=a_{0}, b_{s}=b_{1} y$ inserted in equation (18b) leads to equation (28); for the bending mode $a_{a}=a_{1} y, b_{u}=b_{0}$ inserted in equation (18a) gives equation (29) $\left(a_{0}, a_{1}, b_{0}, b_{1}\right.$ are constants). In each case the remaining boundary condition is satisfied only approximately. For higher-order approximations the differential equations (12) have to be taken into account too.

In the following the superposition of one symmetric and one antisymmetric mode with the same frequency and propagating (to begin with) in the same direction is considered. The displacement at the surface can be represented by

$$
\mathbf{u}(h / 2)=A\left\{p\left(\begin{array}{c}
r_{a} \\
1 \\
0
\end{array}\right) \mathrm{e}^{\mathrm{i} k_{a} x}+(1-p)\left(\begin{array}{c}
1 \\
r_{s}^{-1} \\
0
\end{array}\right) \mathrm{e}^{\mathrm{i}\left(k_{s} x+\phi\right)}\right\} \mathrm{e}^{-\mathrm{i} \omega t},
$$

where the real number $p, 0 \leqslant p \leqslant 1$, is a measure of the portion of antisymmetric mode in the observed wave. The overall (complex) amplitude $A$ and the (real) angle $\phi$ permit normalization of the symmetric and antisymmetric parts inside the curly brackets without loss of generality. The measured displacement ratio $r_{m}$ becomes

$$
r_{m}=\frac{p r_{a}+(1-p) \mathrm{e}^{\mathrm{i} \Phi}}{p+(1-p) r_{s}^{-1} \mathrm{e}^{\mathrm{i} \phi}}
$$

with $\Phi=\left(k_{s}-k_{a}\right) x+\phi$. Solving for $p$ gives

$$
p=1 /\left\{1+\left|r_{s}\right|\left|\frac{r_{m}-r_{a}}{r_{m}-r_{s}}\right|\right\}
$$

the equation for $\Phi$ follows from the fact that $p$ is a real number:

$$
\Phi=\arg \left\{-r_{s}\left(r_{m}-r_{a}\right) /\left(r_{m}-r_{s}\right)\right\}
$$

From $r_{m}$ measurements at various points $x$ the wavenumber difference $k_{s}-k_{a}$ can be verified experimentally, because $\Phi$ is proportional to $x$. The value for $p$, however, should come out independent of $x$; otherwise one would have to consider complex wavenumbers.

The method of decomposition outlined above works if only two modes which have real wavenumbers and different displacement ratios are involved. (The modes may also be both symmetric or both antisymmetric.) With more than two modes a unique decomposition of an observed wave is no longer possible on the basis of the displacement ratios at only one surface of the plate. In practice, one is often interested primarily in the two fundamental modes, which become quasi-longitudinal and bending waves in the limit of low frequencies. Below the lowest cut-off frequency for the higher modes, $f_{c}=c_{t} /(2 h)$ corresponding to $f h / c_{p l}=\sqrt{(1-\sigma) / 8}$, one has indeed only these two modes propagating (provided there are no pure shear waves), and the method is applicable. Real plates, of course, are never infinite as assumed in the calculations here, but it would seem that the determination of $p$ via equation (32) should be a reasonable estimate of the bending component in many cases. 
The propagation direction of the waves is not always known and may even be different for the two modes. In this case an additional measurement of the $z$-component of the displacement is required for an unique decomposition of the observed wave with the more general form

$$
\begin{aligned}
\mathbf{u}(h / 2)= & A\left\{p\left(\begin{array}{c}
r_{a} \cos \phi_{a} \\
1 \\
r_{a} \sin \phi_{a}
\end{array}\right) \mathrm{e}^{\mathrm{i} k_{a}\left(x \cos \phi_{u}+z \sin \phi_{u}\right)}\right. \\
& \left.+(1-p)\left(\begin{array}{c}
\cos \phi_{s} \\
r_{s}^{-1} \\
\sin \phi_{s}
\end{array}\right) \mathrm{e}^{\mathrm{i} k_{s}\left(x \cos \phi_{s}+z \sin \phi_{s}\right)+\mathrm{i} \phi}\right\} \mathrm{e}^{-\mathrm{i} \omega t} .
\end{aligned}
$$

$\phi_{a}$ and $\phi_{s}$ are the angles of the wave vectors of the two modes with the $x$-axis. From the measured displacement ratio $r_{x m}(x$-component to $y$-component $)$ one obtains, for $p$ and $\Phi$,

$$
\begin{gathered}
p=1 /\left\{1+\left|r_{s}\right|\left|\frac{r_{x m}-r_{a} \cos \phi_{a}}{r_{x m}-r_{s} \cos \phi_{s}}\right|\right\}, \\
\Phi=\arg \left\{-r_{s}\left(r_{x m}-r_{a} \cos \phi_{a}\right) /\left(r_{x m}-r_{s} \cos \phi_{s}\right)\right\}
\end{gathered}
$$

with $\Phi=k_{s}\left(x \cos \phi_{s}+z \sin \phi_{s}\right)-k_{a}\left(x \cos \phi_{a}+z \sin \phi_{a}\right)+\phi$.

Similar equations for $p$ and $\Phi$ can be derived with the other measured displacement ratio $r_{z m}$ ( $z$-component to $y$-component). Since these results for $p$ and $\phi$ must agree with those from equations (35) and (36), the real quantities $\phi_{a}$ and $\phi_{s}$ have to satisfy the complex equation

$$
\left(r_{x m}-r_{a} \cos \phi_{a}\right) /\left(r_{x m}-r_{s} \cos \phi_{s}\right)=\left(r_{z m}-r_{a} \sin \phi_{a}\right) /\left(r_{z m}-r_{s} \sin \phi_{s}\right) .
$$

Unfortunately, solving this simple looking equation for $\phi_{a}$ and $\phi_{s}$ is relatively cumbersome. Therefore only an outline can be given of how one can proceed, as follows. Take the imaginary part of equation (37) and solve it (i) for $\cos \phi_{s}$ as a function of $\cos \phi_{a}$ and $\sin \phi_{a}$ (and the various displacement ratios, of course), and (ii) for $\sin \phi_{s}$ in the same way. Then substitute the results for $\cos \phi_{s}$ and $\sin \phi_{s}$ in the real part of equation (37). In general, this resulting equation in $\cos \phi_{a}$ and $\sin \phi_{a}$ will have to be solved numerically.

In the special case where both waves propagate along the same line, but possibly in opposite directions, i.e., $\phi_{a}=\psi$ or $\psi+\pi$ and $\phi_{s}=\psi$ or $\psi+\pi$, unique determination of $\phi_{a}$ and $\phi_{s}$ from equation (37) is no longer possible, because equation (37) always reduces to $\tan \Psi=r_{z m} / r_{x m}$, whereby only the line is specified, not the directions of propagation. Hence $p$ and $\Phi$ exhibit a fourfold ambiguity, which is difficult to remove because displacement ratios in the interior of the plate would have to be known (for instance in the middle plane $y=0$ of the plate, where $r_{a}=r_{s}^{-1}=0$ ). In practice there will be no choice other than evaluating all four possibilities and selecting the one most probable in a particular experimental situation.

If there is only one wave present ( $p=0$ or 1$)$, then the propagation direction can be determined uniquely from $r_{x m}$ and $r_{z m}$, e.g., for $p=1, \cos \phi_{a}=r_{x m} / r_{a}$ and $\sin \phi_{a}=r_{z m} / r_{a}$.

The essence of this exercise on displacement ratios may be summarized as follows. From measurements, which are simple in principle, together with some exact (or almost exact) equations from the fundamental theory of plates, valuable information can be gained without extensive calculations. This experience might stimulate application of fundamental theory to other problems for which one may expect a much more satisfactory treatment without unnecessary approximations. 


\section{ACKNOWLEDGMENT}

The author is grateful to Professor F. P. Mechel for a critical reading of the manuscript. The financial support of this work by the Stifterverband für die Deutsche Wissenschaft is also sincerely acknowledged.

\section{REFERENCES}

1. B. A. Auld 1973 Acoustic Fields and Waves in Solids. Volumes 1 and 2. New York: Wiley.

2. J. D. ACHENBACH 1973 Wave Propagation in Elastic Solids. Amsterdam: North-Holland.

3. T. R. Meeker and A. H. Meitzler 1964 in Physical Acoustics (editor W. P. Mason), Volume 1A. New York: Academic Press. Guided wave propagation in elongated cylinders and plates.

4. R. D. MINDLiN 1960 in Structural Mechanics (editors J. N. Goodier and N. J. Hoff). Oxford: Pergamon Press. Waves and vibrations in isotropic, elastic plates.

5. I. MAlecki 1969 Physical Foundations of Technical Acoustics. Oxford: Pergamon Press.

6. P. M. MORSE and U. INGARD 1968 Theoretical Acoustics. New York: McGraw-Hill.

7. L. CREMER and M. HECKL 1973 Structure-borne sound (translated and revised by E. E. Ungar). Berlin: Springer.

8. F. SAUTER 1948 Zeitschrift für Naturforschung 3a, 548-552. Bemerkungen zur Schwingungstheorie dünner elastischer Platten.

9. S. P. Timoshenko 1921 Philosophical Magazine Series 6, 41, 744-746. On the correction for shear of the differential equation for transverse vibrations of prismatic bars.

10. S. P. Timoshenko 1922 Philosophical Magazine Series 6, 43, 125-131. On the transverse vibrations of bars of uniform cross-section.

11. R. D. MINDLIN 1951 Journal of Applied Mechanics 18, 31-38. Influence of rotatory inertia and shear on flexural motions of isotropic, elastic plates.

12. K. TAMM and O. WEIS 1959 Acustica 9, 275-288. Untersuchungen über periodische Wellen, exponentielle und komplexe Nahfelder im begrenzten Festkörper. 